DOI: $10.12731 / 2306-1561-2013-4-4$

\title{
FORMATION OF SEQUENCE PROGRAM IMPLEMENTATION OF THE STRATEGIC DEVELOPMENT OF INDUSTRIAL ENTERPRISES
}

\author{
Barishnikov A.V., Nikolaev A.B., Zbavitel P.Yu.
}

\begin{abstract}
This paper proposes an approach to the formation of the sequence of the strategic development of industrial enterprises. Integrated management systems and systems are built as a combination of hardware and software information, tools and consumables. The expediency of the use of scheduling for simulation and optimization of the processes of repair and maintenance.
\end{abstract}

Keywords: management, modeling, information environment, scheduling theory, automated control systems.

\section{УДК 681.3}

\section{ФОРМИРОВАНИЕ ПОСЛЕДОВАТЕЛЬНОСТИ ВЫПОЛНЕНИЯ ПРОГРАММЫ СТРАТЕГИЧЕСКОГО РАЗВИТИЯ ПРОМЫШЛЕННЫХ ПРЕДПРИЯТИЙ}

\section{Барышников А.В., Николаев А.Б., Збавитель П.Ю.}

\section{Аннотация}

В статье предложен подход к формированию последовательности выполнения программы стратегического развития промышленных предприятий. Интегральные управленческие комплексы и системы строятся как совокупности программноаппаратных информационных, инструментальных и технологических средств. Показана целесообразность использования теории расписаний для моделирования и оптимизации процессов ремонта и технического обслужсивания.

Ключевые слова: управление, моделирование, информационная среда, теория расписаний, автоматизированные системы управления.

Для кардинального повышения качества деятельности руководителей, представляемые в их распоряжение управленческие комплексы и системы должны обеспечивать эффективную поддержку деятельности в информационной, инструментальной и технологической средах (рисунок 1). 


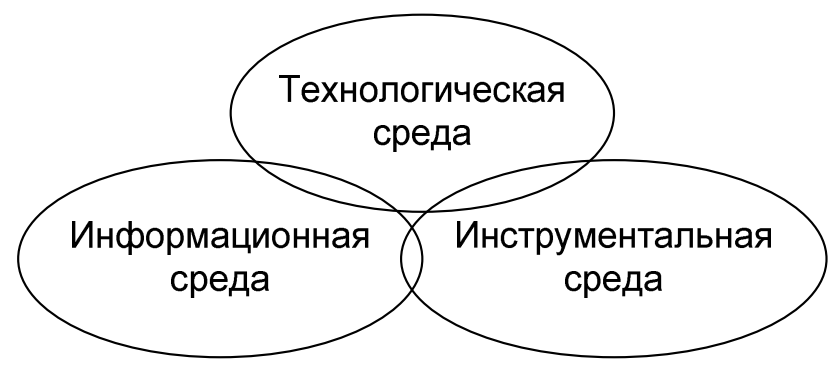

\section{Рисунок 1 - Полнофункциональная среда деятельности руководителя}

С учетом этого интегральные управленческие комплексы и системы строятся как совокупности программно-аппаратных информационных, инструментальных и технологических средств. Макроструктура управленческих систем может быть представлена в следующем виде. Информационная среда поддерживается набором информационных средств $M_{i f}=\left\{m_{i f 1}, m_{i f 2}, \ldots, m_{i f a}\right\}$, инструментальная среда набором инструментальных средств $M_{i s}=\left\{m_{i s 1}, m_{i s 2}, \ldots, m_{i s \beta}\right\}$ и технологическая среда - набором технологических средств $M_{t h}=\left\{m_{t h 1}, m_{t h 2}, \ldots, m_{t h \gamma}\right\}$. Тогда управленческая система образуется при объединении выбранных средств управляющими и информационными линиями связи и описывается как

$$
S_{1}=\left\{M_{1} ; P_{11}^{C}, P_{12}^{C}, \ldots, P_{1 n_{1}}^{C} ; P_{11}^{\prime}, P_{12}^{\prime}, \ldots, P_{1 k_{1}}^{l}\right\}
$$

где M1, - множество средств-носителей; $P_{1 r}^{C}, P_{1 t}^{\prime}$ - заданные на носителях предикаты, определяющие управляющие и информационные связи между средствами.

В общем случае, технологических процесс - последовательность действий (подпроцессов), направленных на получение заданного результата [1 - 5]. Системы управления, построенные на принципах управления функциями, представляют собой иерархическую пирамидальную структуру подразделений, сгруппированных по выполняемым функциям. Под функциональным подразделением можно понимать группу экспертов в данной функциональной области. В организациях, построенных по данному принципу, управление осуществляется на административно-командных принципах. Другим подходом построения систем управления является управление потоками работ или процессами, составляющими деятельность предприятия. Процессное подразделение включает в себя координатора - владельца процесса и исполнителей из различных функциональных областей, сгруппированных по принципу единства результата технологического процесса. Процессный подход позволяет рассматривать деятельность организации как связанную систему технологических процессов, каждый из которых протекает во взаимосвязи с другими или внешней средой. Практика показывает, что система управления, построенная на принципах процессного управления, является более эффективной и результативной по сравнению с равной ей по масштабу функциональной системой. Вместе с тем, разработка и внедрение такой системы - сложный процесс. 
Показана целесообразность использования теории расписаний для моделирования и оптимизации процессов ремонта и технического обслуживания. Кроме того, сформированные модели описания технологических процессов являются непосредственно входными данными для построения оптимальной последовательности этапов ремонтных работ, которая получается на основе формальных моделей теории расписаний.

Проведена классификация задач для реализации ремонтных работ по типу искомого решения:

- задачи упорядочивания. В этих задачах уже задано распределение работ по исполнителям, а также определены все параметры работ (продолжительность выполнения, время поступления и т.д.). Необходимо составить расписание (или порядок) выполнения работ каждым исполнителем;

- задачи согласования. Основное внимание в этих задачах уделяется выбору продолжительности выполнения работ, времени поступления и другим параметрам;

- задачи распределения. Подразумевают поиск оптимального распределения работ по исполнителям.

По типу целевой функции интерес представляют:

- задачи с суммарными критериями оптимизации, в которых необходимо минимизировать суммарное значение моментов окончания обслуживания работ $\sum_{j=1}^{n} C_{j}$

- задачи с минимаксными критериями оптимизации. Отличие этих задач от задач с суммарными критериями заключается в том, что нужно минимизировать максимальное значение критерия

$$
C_{\max }=\max _{j \in N} C_{j}
$$

- многокритериальные задачи оптимизации. Если в исследуемых задачах необходимо построить оптимальное решение с точки зрения нескольких целевых установок (функций), то такие задачи называются многокритериальными. Например, если в упомянутой задаче необходимо не только минимизировать значение $\sum_{j=1}^{n} C_{j}$, но минимизировать и время простоя процессора (прибора), то это многокритериальная задача;

- задачи на построение допустимого расписания. В предыдущем разделе был дан пример такой задачи. Необходимо отметить, что данный класс задач можно свести к оптимизационным задачам, введя специальную функцию штрафа, который нужно минимизировать. Тем не менее, принято выделять такие задачи в отдельный класс.

- задачи упорядочения в теории расписаний рассматриваются при условии, что решены все вопросы, относящиеся к тому, что и каким образом должно 
быть выполнено. Достаточно часто предполагается, что не существует зависимости между характером этих решений и устанавливаемым порядком, т.е. характер работ не зависит от их последовательности выполнения.

Длительность производственного цикла непосредственно влияет на эффективность всего производства. В связи с этим его сокращение является одной из ключевых проблем и всегда включается в программу формирования стратегического плана развития. Если исходной моделью для расчета цикла является сетевая, тогда продолжительность рассчитывается исходя из пути максимальной длины. Рассматривается вопрос снижения длительности цикла на некоторую величину $\Delta$. При этом каждое подразделение объединения готовит и представляет в центр стратегического развития соответствующий комплекс мероприятий по его сокращению.

Пусть $\mathrm{Si}(\tau \mathrm{i})$ - функция затрат реализации i-го этапа, для сокращения цикла на $\tau$ i. Имеют место два варианта решения задачи.

Первый вариант. План мероприятий определяется на основании общей задачи оптимизации:

$$
\sum_{i=1}^{n} S_{i}\left(\tau_{i}\right) \rightarrow \min \sum_{i=1}^{n} \tau_{i}=\Delta
$$

Если найдены оптимальные значения $\tau *$, то подразделение должно сократить длительность своего технологического процесса на i-ом этапе на $\tau^{*}$ i и оно финансируется в объеме $\operatorname{Si}(\tau * \mathrm{i})$.

В отличие от производственного цикла коммерческий цикл включает аспекты продвижения товара от объединения. В данном случае рассматриваются вопросы складирования, транспортировки, продажи, т.е. коммерческий цикл включает все, от чего зависят конечные финансовые показатели. Для коммерческого цикла выбирается путь $\mu$ (для некоторого коммерческого цикла) исходя из критерия оптимизации:

$$
\Phi=\sum_{i \in \mu} s_{i}\left(\tau_{i}\right)+F\left(\sum_{i \in \mu} \tau_{i}\right)_{\rightarrow \text { min. }}
$$

Если имеется сеть, представляющая альтернативные варианты коммерческих

$$
s_{i}\left(\tau_{i}\right)=w_{i} \varphi\left(\frac{\tau_{i}}{w_{i}}\right), \mathrm{i}=1 . . \mathrm{n}, \text { где } \varphi \text {-выпуклая функция } \tau \text {, то }
$$
оптимизация коммерческого цикла решается в два этапа.

Для решения поставленной задачи оптимизации предлагается поисковый алгоритм оптимизации, основанный на походе оптимизации в обстановке шума.

В работе предполагается выполнение следующих условий:

1) Подлежащие выполнению работы определены и известны полностью. Предполагается, что все заданные работы должны быть выполнены (разбиение совокупности работ на классы выполняемых и невыполняемых не входит в задачу упорядочения). 
2) Однозначно определены ресурсы, выделяемые для выполнения заданных работ.

3) Задана совокупность всех элементарных действий, связанных с выполнением каждой из работ, и ограничений, налагаемых на порядок их выполнения. Известно также, каким образом осуществляются эти действия, и что существует, по крайней мере, по одному устройству, способному выполнить каждое из них.

Предположим, что исходные работы разбиты на $\mathrm{k}$ групп с числом работ $n_{i}, i=1, k$ в каждой группе. Предположим также, что состав групп и очередность работ в группе фиксированы и что переход к следующей группе возможен только после того, как выполнены все работы предыдущей. Требуется выбрать один из возможных способов упорядочения групп.

Пусть tij - длительность выполнения работы j в группе i, Fij - длительность прохождения работы ј в группе $\mathrm{i}, \quad t^{\prime}{ }_{i}=\sum_{j=1}^{n_{i}} t_{i j} \quad$ - общая длительность всех работ і-ой группы, Fij'=Fi,nj - длительность прохождения i-ой группы, равная длительности прохождения последней работы в ней.

В качестве критерия может выступать минимум средней длительности прохождения группы. $\overline{F^{\prime}}=\sum_{i=1}^{k} F^{\prime}{ }_{i} / k$. При этом каждую группу нужно рассматривать как работу длительностью t'i и применить алгоритм упорядочения в соответствии с минимальной длительностью. Это означает, что группы упорядочиваются так, что: $t_{[1]}^{\prime} \leq t_{[2]}^{\prime} \leq \ldots \leq t_{[k]}^{\prime}$.

При минимизации взвешенной средней длительности прохождения группы $\overline{F^{\prime}}=\sum_{i=1}^{k} n_{i} F^{\prime} / n$

$$
t_{[1]}^{\prime} / n_{[1]} \leq t_{[2]}^{\prime} / n_{[2]} \leq \ldots \leq t_{[k]}^{\prime} / n_{[k]} \text {. }
$$

Далее в работе рассмотрен случай, когда $\mathrm{n}$ работ разбито на $\mathrm{k}$ цепочек. При этом отношения предшествования определяют для каждой работы не более одной предшествующей и не более одной последующей, например,

$$
\text { (a) I (b) } \rightarrow \text { (c) / (d) } \rightarrow \text { (e) } \rightarrow(f) \quad /(g) \rightarrow(h)
$$

При наличии таких ограничений, упорядочение, минимизирующее среднюю длительность прохождения, определяется следующим образом. Пусть для системы множество работ разбивается на $\mathrm{k}$ непересекающихся цепочек, внутри каждой из которых задана последовательность выполнения работ, причем допускается прерывание цепочек. Тогда средняя длительность прохождения минимизируется упорядочением, устанавливаемым следующим образом:

1) Для каждой работы ј в і-й цепочке вычисляется средняя длительность работ подцепочки, состоящей из первых ј работ - $I_{i j}=\sum_{r=1}^{j} t_{i r} / j$. 
2) Для каждой цепочки вычисляется $L_{i}\left(r_{i}\right)=\min \left(l_{i 1}, l_{i 2}, \ldots, l_{i n_{i}}\right)=I_{i r_{i}}$, где ri - второй индекс минимальной из величин $l_{i 1}, l_{i 2}, \ldots, l_{i n_{i}}$.

3) Выбирается такая цепочка I, что $L_{l}\left(r_{l}\right) \leq L_{i}\left(r_{i}\right)$ для всех і и первые ri работ в цепочке I формируют начало очередности выполнения работ.

4) Вычисляются заново величины $I_{i j}, L_{i}$ без учета первых ri работ цепочки I.

Третий и четвертый шаги повторяются до тех пор, пока не будут упорядочены все работы.

Предложенный алгоритм позволяет упорядочить работы с более общим видом ограничений (рисунок 2), задаваемых отношением предшествования.

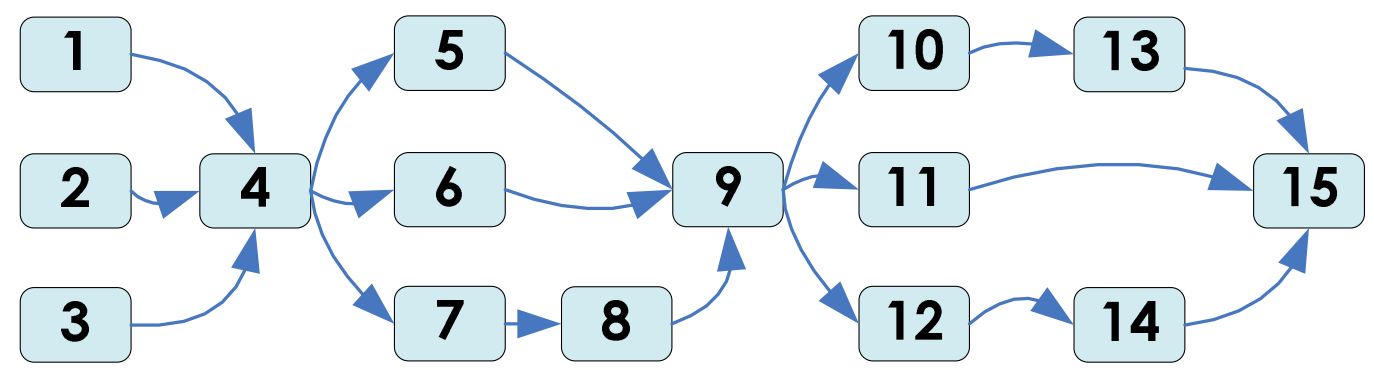

Рисунок 2 - Отношение предшествования работ

В этом графе алгоритм можно применить к совокупности работ $\{1,2,3\},\{5,6,7,8\}$ поскольку они образуют независимые последовательности. Формирование результирующего расписания таково: упорядочение работ $\{1,2,3\}$; назначение узловой работы 4; упорядочение работ $\{5,6,7,8\}$; назначение узловой работы 9; упорядочение работ $\{10,11,12,13,14\}$; назначение узловой работы 15.

Например, стоит задача упорядочения совокупности работ, представленных в таблице 1.

\section{Таблица 1 - Параметры работ в цепочках}

\begin{tabular}{|c|c|c|c|c|}
\hline цепочка 1 & $1.1 / 3$ & $1.2 / 4$ & & \\
\hline цепочка 2 & $2.1 / 1$ & $2.2 / 2$ & $2.3 / 5$ & $2.4 / 2$ \\
\hline цепочка 3 & $3.1 / 6$ & $3.2 / 2$ & $3.3 / 3$ & \\
\hline
\end{tabular}

В таблице принято обозначение: i.j/tij , где i - номер цепочки, j - номер работы в цепочке, tij - длительность. В результате работы алгоритма (на основании четырех итераций) получим значения средних длительностей работ (таблица 2.) для всех цепочек 


\section{Таблица 2 - Средние длительности работ подцепочек}

Итерация 4.

\begin{tabular}{|c|c|c|c|c|c|}
\hline цепочка 1 & $112=4$ & & & 4 & \\
\hline цепочка 2 & $123=5$ & $124=3.5$ & & 3.5 & 3.5 \\
\hline цепочка 3 & $131=6$ & $132=4$ & $133=3.66$ & 3.66 & \\
\hline
\end{tabular}

В результате, оптимальная последовательность выполнения работ будет следующей $\mathrm{S}=\{2.1,2.2,1.1,2.3,2.4,3.1,3.2,3.3,1.2\}$.

\section{Список информационных источников}

[1] Барышников А.В., Чернявский А., Борщ В., Моисеев А. Методика оптимизации предупредительных замен в задаче планирования производственного цикла ремонтного предприятия // Наука и образование. МГТУ им. Н.Э.Баумана. Электрон. журн. 2013. № 8. DOI: 10.7463/0813.0615305.

[2] Барышников А.В., Чернявский А., Борщ В. Статистическое моделирование поставок комплектующих в сети ремонтных предприятий автомобильной промышленности // Наука и образование. МГТУ им. Н.Э. Баумана. Электрон. журн. 2013. № 9. DOI: 10.7463/0913.0615319.

[3] Николаев А.Б. Информационные технологии в менеджменте и транспортной логистике: учебное пособие / А.Б. Николаев, А.В. Остроух. - Saint-Louis, MO, USA: Publishing House Science and Innovation Center, 2013. - 254 c. - ISBN 978-0615-67110-9.

[4] Остроух, А.В. Информационные технологии в научной и производственной деятельности / [ред. А.В. Остроух] - М: ООО "Техполиграфцентр", 2011. - 240 с. ISBN 978-5-94385-056-1.

[5] Суркова Н.Е., Угарова Ю.Н. Организация работы ит-подразделения в холдинговых структурах // Автоматизация и управление в технических системах. - 2013. - № 1(3); URL: auts.esrae.ru/3-59 (дата обращения: 31.10.2013). 